\title{
MiR-448 suppresses cell proliferation and glycolysis of hepatocellular carcinoma through inhibiting IGF-1R expression
}

\author{
Yilang Wang ${ }^{1,2 \#}$, Xiaohong Chen ${ }^{3 \#}$, Ninghua Yao ${ }^{4 \#}$, Jun Gong ${ }^{2}$, Yongfeng $\mathrm{Cao}^{2}$, Xiaoqing Su${ }^{2}, \mathrm{Xiu}^{\mathrm{Feng}}{ }^{4}$, \\ Min Tao ${ }^{1,5}$ \\ ${ }^{1}$ Department of Oncology, The First Affiliated Hospital of Soochow University, Suzhou, China; ${ }^{2}$ Department of Medical Oncology, The Affiliated \\ Tumor Hospital of Nantong University, Nantong, China; ${ }^{3}$ Department of Ultrasound Medicine, The Second Affiliated Hospital of Nantong \\ University, Nantong, China; ${ }^{4}$ Department of Oncology, Affiliated Hospital of Nantong University, Nantong, China; ${ }^{5}$ Department of Oncology, \\ Dushu Lake Hospital Affiliated to Soochow University, Suzhou, China \\ Contributions: (I) Conception and design: M Tao; (II) Administrative support: M Tao, N Yao, Y Cao; (III) Provision of study materials or patients: Y \\ Wang, X Chen, J Gong, X Su; (IV) Collection and assembly of data: Y Wang, X Feng; (V) Data analysis and interpretation: Y Wang; (VI) Manuscript \\ writing: All authors; (VII) Final approval of manuscript: All authors. \\ "These authors contributed equally to this work. \\ Correspondence to: Min Tao. Department of Oncology, The First Affiliated Hospital of Soochow University, Suzhou, China. Email: taomin@suda.edu.cn.
}

Background: Microribonucleic acids (miRNAs) have been shown to play important roles in hepatocellular carcinoma (HCC) progression. MiR-448 has frequently been shown to be a tumor suppressor, and is abnormally expressed in HCC tumor tissues. However, little is known about the role of miR-448 in HCC development. In this article, the regulatory role of miR-448 on insulin-like growth factor 1 receptor (IGF-1R) in modulating hepatoma cell viability and glycolysis was investigated.

Methods: The expression of miR-448 profiles in clinical tumor tissues and cell lines was examined using quantitative real-time polymerase chain reaction (qRT-PCR). HepG2 and Huh7 cells were transfected with miR-448 mimics, inhibitors, and scramble sequences. Cell viability and apoptosis were determined by a Cell Counting Kit- 8 assay and a flow cytometry analysis. IGF-1R, a potential target of miR-448, was selected following a bioinformatic analysis, and the regulatory effects of miR-448 on IGF-1R expression was confirmed by luciferase reporter assay, qRT-PCR, and western blot. Glucose uptake, lactate production, and adenosine triphosphate (ATP) generation were detected by corresponding kits.

Results: Decreased miR-448 expression was observed in both HCC patients' tumor tissues and hepatoma cells in vitro. The overexpression of miR-448 in HepG2 and Huh7 cells decreased cell viability and increased apoptosis. Additionally, the overexpression of miR-448 or the knockdown of IGF-1R lowered the level of glucose uptake, lactate production, and ATP generation, while the knockdown of miR-448 increased glycolysis. Further, aberrantly expressed miR-448 downregulated IGF-1R levels, while the inhibition of miR448 resulted in the upregulation of IGF-1R in both HepG2 and Huh7 cells. In addition, miR-448 interacted with the wild-type 3 'untranslated regions (3'UTRs) of IGF-1R, but had no effect on the mutant 3'UTRs. The expression of IGF-1R was increased in HCC patients' tumor tissues and serum, and was inversely correlated with miR-448 expression.

Conclusions: The increased expression of miR-448 appears to downregulate the expression of IGF-1R by interacting with the 3'UTR in HCC progression. These findings highlight its role as a potential target for HCC therapy.

Keywords: Hepatocellular carcinoma (HCC); miR-448; insulin-like growth factor 1 receptor (IGF-1R); apoptosis; glycolysis

Submitted Aug 06, 2021. Accepted for publication Feb 18, 2022.

doi: 10.21037/jgo-22-90

View this article at: https://dx.doi.org/10.21037/jgo-22-90 


\section{Introduction}

Due to its very high morbidity and mortality rates, hepatocellular carcinoma (HCC) is one of the most prevalent malignant tumors (1). It has the 5 th highest incidence and is the 3 rd leading cause of cancer-related deaths among cancers, and about $80 \%$ of all primary liver cancers are HCC in China $(2,3)$. Due to a lack of effective diagnostic biomarkers and obvious symptoms in the early stages of HCC, most patients suffering from liver cancer are only diagnosed at an advanced stage with malignant metastases (4). Newly developed medical treatments, such as transplantation, chemotherapy, radiotherapy, immunotherapy, and targeted therapy, partially improve the prospects of HCC patients for whom prognosis is bleak $(5,6)$. Thus, extra biomarkers and underlying mechanisms urgently need to be identified to monitor the progression of HCC.

Micro ribonucleic acids (miRNAs) are a type of endogenous, small, non-coding ribonucleic acids (RNAs) that have critical roles in manipulating the expression of target genes (7). Clinical cancer investigations have shown that many miRNAs function as biomarkers for diagnosis, therapy, and prognosis (8). In former studies, miR-448 was reported to be a fundamental cancer-related miRNA in many cancers, including colon cancer (9), lung cancer (10), pituitary adenoma cells (11), pancreatic cancer (12), oral squamous cell carcinoma (13), breast cancer (14), bladder cancer (15), gastric cancer (16), and HCC (17). Especially, among reports above, miR-448 was found to be decrease in HCC samples and influence cell invasion and epithelialmesenchymal transition (EMT). However, the typical role of miR-448 in the progression and metabolism of HCC requires further investigation.

Cell metabolism is widely reported to be involved in the development of HCC, and has an important role in diverting the energy supply source that shapes the metabolic microenvironment (18). Metabolism reprogram was important in regulating cell viability, cell division, cell differentiation and cell migration. During tumorigenesis, cells was prior to perform oxidative glycolysis by converting the glucose into lactate, even under the environment full of oxygen. Through so called "Warburg effect", the rate of glucose absorption, consumption and lactate reproduction was enhanced, which rapidly supplied with the tumor cells with plenty of adenosine triphosphate (ATP), nuclear acids, lipids and amino acids et al. Additionally, the acidic circumstance maintained by the production of lactate that shaped the tumor microenvironment and encouraged the invasion, migration and immune response. "Warburg effect" was paid more attention in HCC investigation while the potential association between it and miR-448 need extra study. Insulin signaling cascade is identified as one of the dominant pathways that manipulates the use of cell energy $(19,20)$. Insulin-like growth factor 1 receptor (IGF-1R) is a key factor involved in these pathways. It is defined as a transmembrane protein that contains a tyrosine-kinase domain and ligand-binding domain (21). The gene is about $100 \mathrm{~kb}$ in length that harbors 21 exons. Complex of IGF-1R was consisted with two equal $\alpha$-subunits and identical $\beta$-subunits. Mechanistically, the $\alpha$-subunits activate the $\beta$-subunits by autophosphorylation of their tyrosine residues. The interaction between IGF-1R and its ligand, insulin-like growth factor 1 (IGF-1), ignites phosphatidylinositol-3-kinase and protein kinase B signaling cascades and other cascades (22). Cumulative evidence has shown that IGF-1R and other related signaling pathways are involved in the development of HCC (23). Elevated levels of IGF-1R in HCC patients may result in cancer cell proliferation and are related to poor prognosis (24). In addition, IGF-1R was reported to be involved in cancer cell-related glycolysis, which facilitates glucose uptake, lactate production, and ATP production (22). Thus, we sought to confirm the function of miR-448 and IGF-1R in HCC. We identified expression patterns of miR-448 and IGF-1R in clinical HCC patients and variations in survival associated with these patterns in vivo and in vitro. Further, the regulatory relationship between miR-448 and IGF$1 \mathrm{R}$ were investigated in progression of HCC. Thus, the activation of miR-448 might provide a novel way of curing HCC. We present the following article in accordance with the MDAR reporting checklist (available at https://jgo. amegroups.com/article/view/10.21037/jgo-22-90/rc).

\section{Methods}

\section{Clinical samples}

In total 82 pairs of HCC patients were enrolled in this study. Tumor tissues and adjacent normal tissues were harvested by surgery at The Affiliated Tumor Hospital of Nantong University from January 2017 to November 2018. 
Table 1 Correlation between clinicopathological characteristics and miR-448 expression in 82 hepatocellular carcinoma patients by Fisher's exact test analysis

\begin{tabular}{|c|c|c|c|c|}
\hline \multirow{2}{*}{ Variables } & \multirow{2}{*}{$\begin{array}{l}\text { No. of } \\
\text { cases }\end{array}$} & \multicolumn{2}{|c|}{ miR-448 expression*, n (\%) } & \multirow{2}{*}{$P$ value } \\
\hline & & Low & High & \\
\hline \multicolumn{4}{|l|}{ Gender } & 0.327 \\
\hline Male & 68 & $37(54.4)$ & $31(45.6)$ & \\
\hline Female & 14 & $5(35.7)$ & $9(64.3)$ & \\
\hline \multicolumn{4}{|l|}{ Age (years) } & 0.11 \\
\hline$<50$ & 35 & $22(62.9)$ & $13(37.1)$ & \\
\hline$\geq 50$ & 47 & $20(42.6)$ & $27(57.4)$ & \\
\hline \multicolumn{4}{|l|}{ HBV } & 0.311 \\
\hline Absent & 24 & $10(41.7)$ & 14 (58.3) & \\
\hline Present & 58 & $33(56.9)$ & $25(43.1)$ & \\
\hline \multicolumn{4}{|c|}{ Tumor size $(\mathrm{cm})$} & 0.023 \\
\hline$<5$ & 31 & $10(32.3)$ & $21(67.7)$ & \\
\hline$\geq 5$ & 51 & $31(60.8)$ & 20 (39.2) & \\
\hline \multicolumn{4}{|c|}{ TNM tumor stage } & 0.022 \\
\hline $\mathrm{I}+\mathrm{II}$ & 64 & $28(43.8)$ & $36(56.3)$ & \\
\hline III & 18 & $14(77.8)$ & $4(22.2)$ & \\
\hline \multicolumn{4}{|l|}{ Cirrhosis } & 0.501 \\
\hline Absent & 34 & $15(44.1)$ & 19 (55.9) & \\
\hline Present & 48 & $26(54.2)$ & $22(45.8)$ & \\
\hline \multicolumn{4}{|l|}{$\operatorname{AFP}(n g / m L)$} & 0.89 \\
\hline$<20$ & 23 & $12(52.2)$ & $11(47.8)$ & \\
\hline$\geq 20$ & 59 & $30(50.8)$ & $29(49.2)$ & \\
\hline
\end{tabular}

*, miR-448 expression that increased over 2-fold in the caner tissues compared with relevant adjacent tissues designates as "High" group. AFP, alpha-fetoprotein.

None of the patients underwent any extra treatment before surgery. Samples were washed with pre-cold saline and then frozen in liquid nitrogen before being analyzed. All the patients signed informed consent form. The present study was approved by the Ethics Committee of The Affiliated Tumor Hospital of Nantong University (ethic review No. LW2016-005), and the clinical investigation was conducted in accordance with the Declaration of Helsinki (as revised in 2013). Information about patients is set out in Table 1.

\section{Survival rate investigation by Kaplan-Meier (K-M) analysis}

The prognostic value of IGF-1R in HCC between the high- and low-gene expression groups was assessed by a Kaplan-Meier (K-M) analysis and log-rank test. All data were acquired from Kaplan-Meier Plotter database (http:// www.kmplot.com/). The overall survival (OS) analysis was generated using the clinical data of 160 patients with high IGF-1R expression, and 539 patients with low IGF-1R expression based on the optimal thresholds. Meanwhile, the disease-free survival (DFS) analysis was plotted with data from 159 patients of high-expressed IGF-1R and 340 patients of low-expressed IGF-1R.

The prognostic value of miR-448 in HCC between the high- and low-expression groups was tested by K-M plotter and log-rank test. All data were acquired from KaplanMeier Plotter database (http://www.kmplot.com/). The OS analyses were derived from the clinical data of 267 patients with low miR-448 expression and 83 patients with high miR-448 expression. Data from 237 patients with low miR-448 expression and 68 patients with high miR-448 expression were taken to generate the DFS plot.

\section{Cell culture and transfection}

The LO2, Hep3B, Bel-7404, Huh7 and HepG2 cell lines (Cell Bank of Chinese Academy of Sciences, Shanghai, China) were cultured in Dulbecco's Modified Eagle Medium (Gibco, NY, USA) containing 10\% fetal bovine serum (Gibco, NY, USA), and incubated at $37{ }^{\circ} \mathrm{C}$ with $5 \%$ carbon dioxide. The miR-448 mimics, miR-448 inhibitors, and their corresponding controls were designed and provided by the RiboBio Company (Guangzhou, China). The small interfering RNA (siRNA) for IGF-1R was also synthesized by RiboBio and which targeted on the site of GUUCCUU at 3'-UTR. The cells were transfected with oligonucleotides $(25 \mathrm{nM}$ ) for $48 \mathrm{~h}$ using Lipofectamine 2000 (Thermo Fisher Scientific, USA). IGF-1R siRNA was transfected at a $20 \mathrm{nM}$ concentration for $24 \mathrm{~h}$.

\section{Cell proliferation assay}

The cells were pre-seeded in 96-well plates containing about $2 \times 10^{3}$ cells per well. Appropriate miRNA fragments were transferred to HepG2 and Huh7 cells for $24 \mathrm{~h}$, 
after which $10 \mu \mathrm{L}$ Cell Counting Kit 8 (CCK-8) solution (Dojindo Molecular Technology, Kyushu, Japan) was added to each well and the cells were incubated at $37{ }^{\circ} \mathrm{C}$ for $4 \mathrm{~h}$. The absorbance was monitored by a microplate reader (BioRad laboratories, Hercules, CA, USA) at $450 \mathrm{~nm}$. The data were collected as 3 replicates.

\section{Flow cytometry}

The cells were seeded and transfected as described above, and then collected for flow cytometry to determine the apoptosis rate. The cells were washed and then fixed with pre-cooled ethyl alcohol for $20 \mathrm{~min}$ at room temperature. The cells were washed with phosphate-buffered saline (PBS) and then suspended with binding buffer. The density of the cells was adjusted to $1 \times 10^{5}$ cells $/ \mathrm{mL}$ and $100 \mu \mathrm{L}$ was allocated to each tube. Then, $5 \mu \mathrm{L}$ Annexin V- fluorescein isothiocyanate and $5 \mu \mathrm{L}$ propidium iodide was added to the tubes, and the cells were incubated for $15 \mathrm{~min}$ in the dark. Additionally, $400 \mu \mathrm{L}$ binding buffer was added to dilute the reaction buffer, and flow cytometer analysis fluorescenceactivated cell sorting (FACS; Beckman Coulter, Pasadena, CA, USA) was then conducted. The data were measured in triplicate.

\section{RT-PCR analysis}

The total RNA from cells was isolated with guanidinium thiocyanate-phenol-chloroform extraction buffer (Vazyme, Nanjing, China). For the messenger RNA (mRNA) analysis, the complementary deoxyribonucleic acids (cDNAs) were generated with $1 \mu \mathrm{g}$ RNA by a package of QRT SuperMix for quantitative real-time polymerase chain reaction (qPCR; Vazyme, Nanjing, China). The templates were analyzed by Stepone Plus (Applied Biosystems, IL, USA). The primers were designed and produced by Genescript Biotech (Nanjing, China), and glyceraldehyde 3-phosphate dehydrogenase (GAPDH) was used as the internal control. The following genes as below were investigated together with each amplified primer sequences: IGF-1R primers forward: 5'-GAGGAGATGGAGCCTGGCTTC-3', reverse: 5'-GTGTCTGTCGGGCAGTGGCAG-3'. GAPDH primers forward: 5'-GCAATGTTGCCAGTGTCTGT-3', and reverse: 5'-GCCTTGACCTTTTCAGCAAG-3'.

For the miRNA analysis, the miRNA 1st Strand cDNA
Synthesis Kit (Vazyme, Nanjing, China) was used to prepare the cDNAs, and the miRNA Universal SYBR qPCR Master Mix (Vazyme, Nanjing, China) was used to analyze the miRNAs. The following primer sequences were used: miR448 forward: 5'-TTATTGCGATGTGTTCCTTATG-3', and reverse: 5'-ATGCATGCCACGGGCATATACACT-3'. The following internal control primers were used: U6 forward: 5'-CGCGCTTCGGCAGCACATATACT-3', and reverse: 5'-ACGCTTCACGAATTTGCGTGTC-3'. The relative expression of mRNA or miRNA was evaluated using the $2^{-\Delta \Delta C T}$ (Cycle threshold) method.

\section{Western blot}

The cells were harvested and washed twice with pre-cooled PBS, and then completely digested in radioimmunoprecipitation assay lysis buffer (Epizyme, Shanghai, China) mixed with phosphatase and proteinase inhibitor cocktail (Epizyme, Shanghai, China). The isolated protein samples were adjusted to the same concentration and denatured in boiling water for $10 \mathrm{~min}$. Sodium dodecyl sulphate-polyacrylamide gel electrophoresis was used to separate the targeted proteins and then they were transferred to polyvinylidene fluoride membranes (Millipore, Billerica, USA). The blocked membranes were incubated with certain primary antibodies overnight at $4{ }^{\circ} \mathrm{C}$. Anti-IGF-1R (154 kDa, ab131476, Abcam, MA, USA) was diluted at 1:500; anti-GAPDH (ab181602, Abcam, MA, USA) was diluted at 1:2,000. Bands were detected by Tanon 5200 systems (Shanghai, China) with an enhanced ECL (Electrochemiluminescence) Super Signal (Pierce, Rockford, IL, USA). The density of each band was analyzed by ImageJ software (NIH, NY, USA), and GAPDH was used as the control. The data were gathered in triplicates.

\section{Glucose uptake, lactate production, and ATP generation assay}

To determine the glucose uptake, lactate production, and ATP generation, the cells were seeded in 96-well plates and deprived from glucose supply by culturing with $100 \mu \mathrm{L}$ of Krebs-Ringer-Phosphate-HEPES buffer containing 2\% BSA (bovine serum albumin) for $40 \mathrm{~min}$. The cells were then incubated with $10 \mu \mathrm{L} 2$-DG $(10 \mathrm{mM})$ for $20 \mathrm{~min}$. The cells were gathered and analyzed according to the instructions of Glucose Uptake Colorimetric Assay kit 
(BioVision, Milpitas, CA, USA). Extra $5 \times 10^{5}$ cells were harvested and homogenized in $50 \mu \mathrm{L}$ lysis buffer in accordance with the manufacturer's instructions. The mixture was centrifugated and the supernatant was then assayed with a Lactate Assay Kit II, and an ATP Colorimetric Assay kit (BioVision, Milpitas, CA, USA).

\section{Luciferase reporter assay}

The cells were cultured in 24-well plates and were transfected with a single sequence or a mixture of wild-type or mutated pGL3-IGF-1R-3'untranslated regions (3'UTRs) and miR-448 scramble fragments or miR-448 mimic segments using the Lipofectamine 2000 (Thermo Fisher Scientific. USA) in accordance with the manufacturer's instructions. Renilla and firefly luciferase signals were detected using the dual-luciferase reporter assay system (Promega, Madison, WI, USA) in accordance with the manufacturer's instructions.

\section{Statistical analyses}

All the data in this study are presented as mean \pm standard deviation (SD). The statistical significance of the cell proliferation, expression, glucose uptake, lactate, ATP, and the luciferase reporter assay results were determined with the 2-tailed student's t-test. The OS and DFS between the low- and high-gene expression groups were compared with log-rank tests. The correlation between the IGF-1R mRNA expression and the miR-448 mRNA expression was evaluated by a linear regression analysis. SPSS 24.0 software, GraphPad 8.0 software, and ImageJ software were used in the statistical analyses. A 2-talied $\mathrm{p}$ value less than 0.05 was considered statistically significant.

\section{Results}

\section{The expression of miR-448 is decreased in human HCC tissues and cell lines}

To investigate the potential role of miR-448 in HCC, the expression of miR-448 in 82 HCC tissues and their adjacent liver tissues was assessed by quantitative real-time polymerase chain reaction (qRT-PCR). The expression of miR-448 was more downregulated in HCC tumors than normal liver tissues (see Figure 1A). In addition, the expression of miR-448 in HCC cell lines and LO2 normal liver cells were examined by qRT-PCR, and the results showed a significant decrease in miR-448 expression in the Hep3B, Bel-7404, Huh7, and HepG2 cells compared to the $\mathrm{LO} 2$ cells, which was similar to the expression pattern in HCC tissues (see Figure 1B). Among the HCC cell lines, HepG2 exhibited the lowest expression level of miR448, and Huh7 displayed the highest expression level (see Figure 1B); thus, they were chosen as the cell models in this study.

To clarify the potential clinicopathological significance of miR-448 levels, transcriptional variations were analyzed. The results of this analysis are set out in Table 1. The decreased expression of miR-448 was significantly associated with both large tumor size $(\mathrm{P}<0.05)$ and a more advanced stage $(\mathrm{P}<0.05)$, but was not corelated with gender, age, HBV (Hepatitis B), cirrhosis, and AFP (Alpha fetoprotein) level. These results indicate that miR-448 acts as an inhibitor in HCC.

To further determine the prognostic relevance of miR448 expression, data mining of the K-M plotter database and log-rank tests were conducted to assess the relationship between HCC patient survival rate and miR-448 expression. The results revealed that high levels of miR-448 expression were corelated with a markedly poorer prognosis (see Figure $1 C, 1 D$ ). This suggests that miR-448 is related to HCC progression.

\section{MiR-448 promotes cell apoptosis in HCC cells}

To evaluate the function of miR-448 in the growth of HCC, HepG1 and Huh7 cells were ectopically overexpressed by transfection of miR-448 mimic and corresponding scramble sequences. The transcriptional expression of miR-448 was increased after transfection (see Figure 2A). The overexpression of miR-448 significantly decreased the viability of HepG2 and Huh7 cells (see Figure 2B,2C). Similarly, the results of the FACS assay demonstrated that cell apoptosis was increased in HepG2 (see Figure 2D,2E) and Huh7 (see Figure 2F-2H) cells under the condition of miR-448 overexpression.

\section{MiR-448 directly targets IGF-1R in HCC}

To investigate the molecular mechanism of miR-448 in regulating HCC progression, TargetScan, PicTar, and miRanda databases were used to identify potential 
A

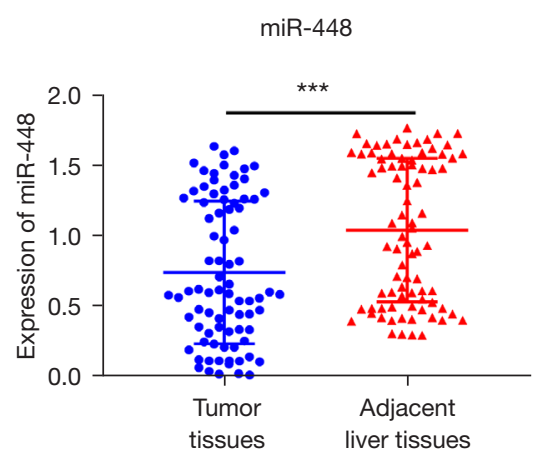

C

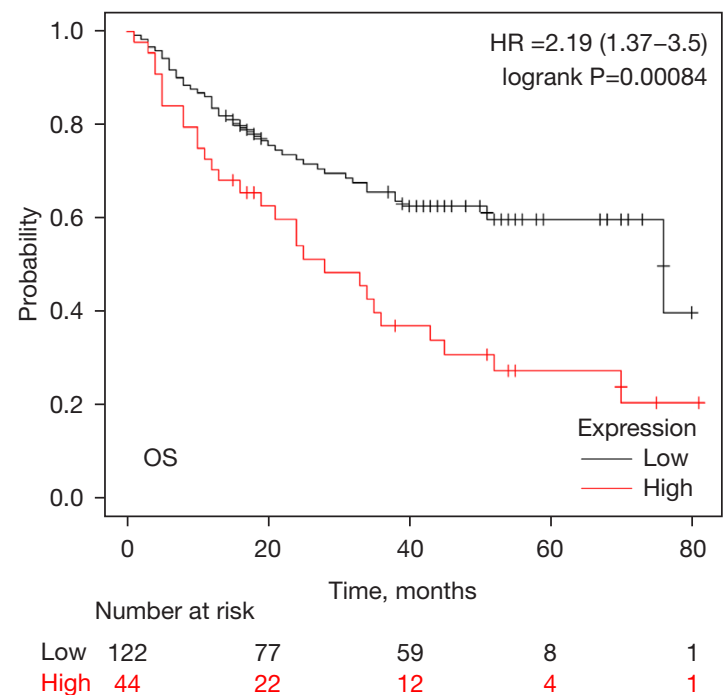

B

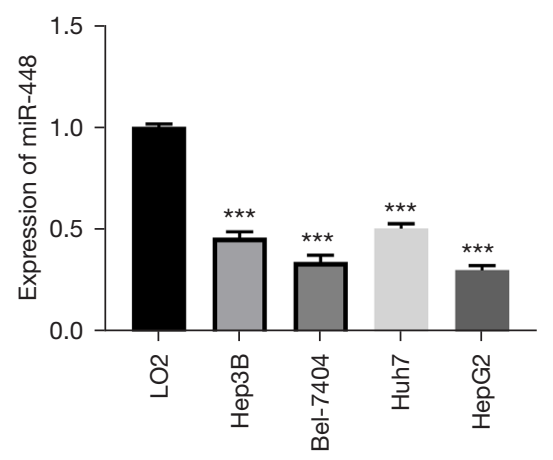

D hsa-miR-448

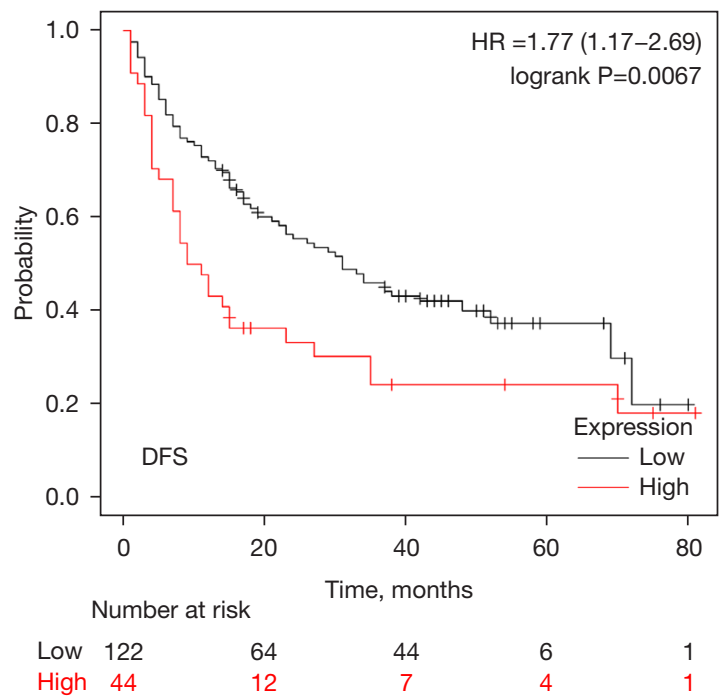

Figure 1 Reduced expression of miR-448 in hepatocellular carcinoma (HCC) tissues and cells. (A) The expression of miR-448 was evaluated in 82 pairs of HCC tumor tissues and adjacent liver tissues. (B) miR-448 content in the Hep3B, Bel-7404, Huh7, and HepG2 cell lines and the control LO2 line was assessed via qRT-PCR. N=3. (C,D) Correlations between miR-448 and HCC patients' overall survival (OS) and disease-free survival (DFS), as compared by log-rank tests. ${ }^{* * *}, \mathrm{P}<0.001$.

targets of miR-448. A bioinformatics analysis was used to identify IGF-1R as a potential target among hundreds of different predicted ones. To further assess the interaction between miR-448 and IGF-1R 3'UTR in HCC, wildtype and mutant 3'UTRs of IGF-1R were subcloned into the luciferase reporter plasmids (see Figure 3A). Mir-448 transfection significantly inhibited the luciferase activities, while antagomir adoption increased luminance density in both HepG2 (see Figure 3B) and Huh7 (see Figure 3C) cells. The wild-type IGF-1R 3'UTR, including a luciferase reporter vector, exhibited decreased activities following miR-448 mimic treatment compared to the backbone vector (see Figure 3D,3E). However, no effects were observed in the mutated IGF-1R 3'UTR transfused cells. Together, these results indicate that IGF-1R is a direct target of miR-448.

\section{MiR-448 affects HCC cell glycolysis}

The IGF-1R is a key regulator in energy metabolism, 
A
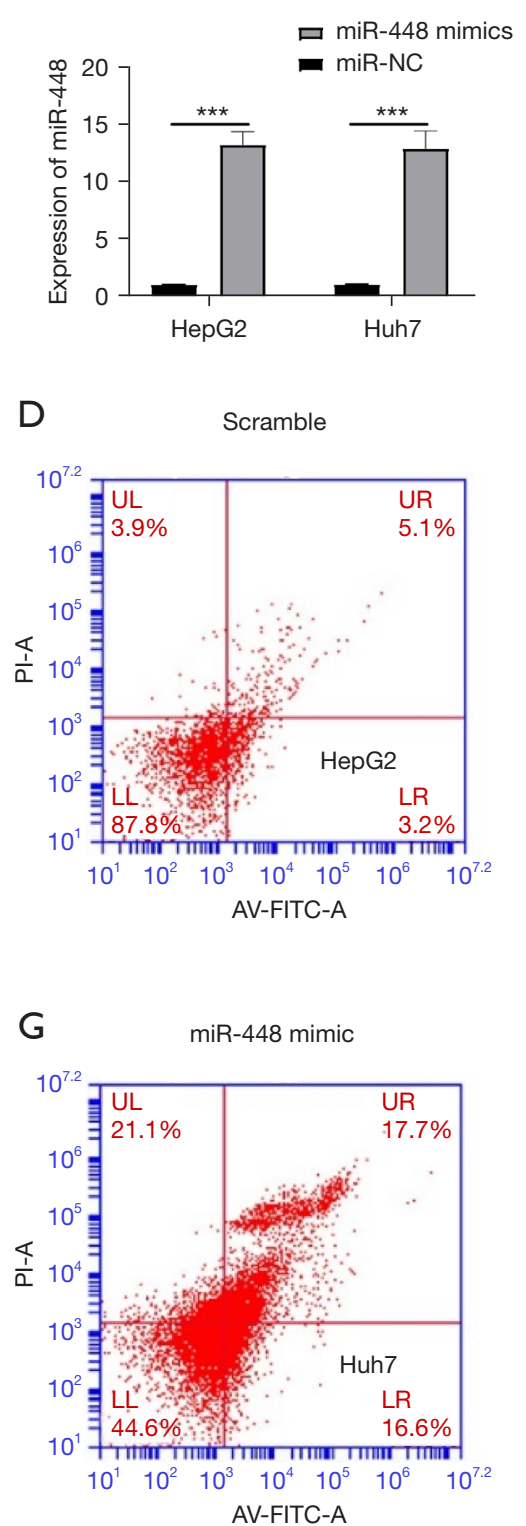

B

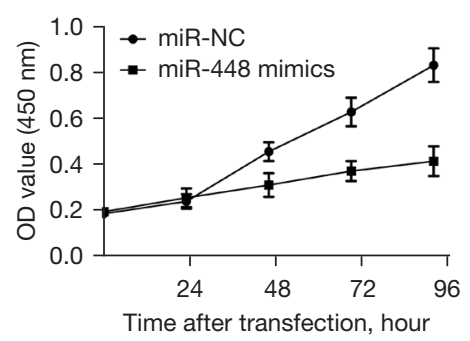

$\mathrm{E}$

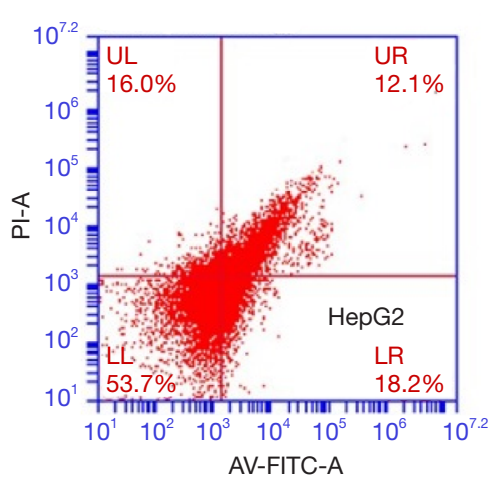

$\mathrm{H}$

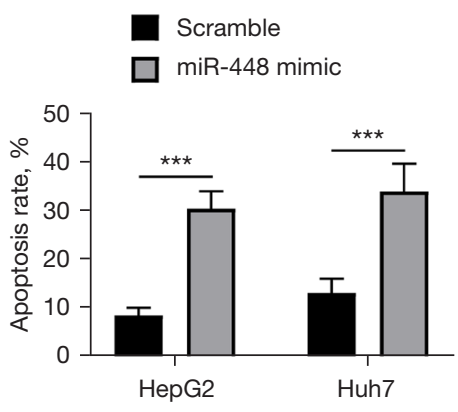

C

Cell viability of Huh7

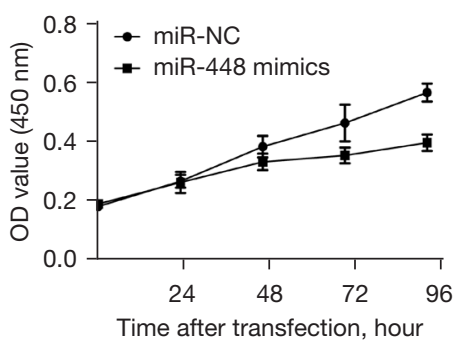

$\mathrm{F}$

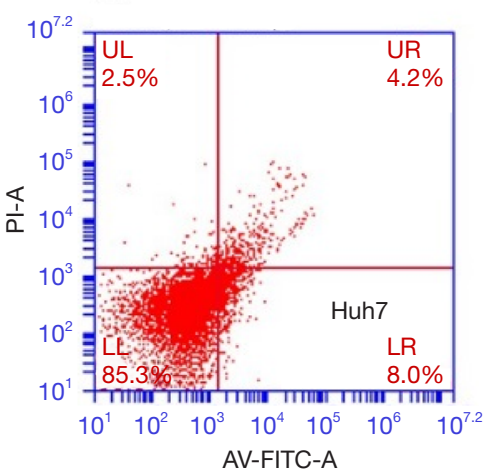

Figure 2 MiR-448 over expression increases hepatocellular carcinoma (HCC) apoptosis. (A) The relative expression of miR-448 in HepG2 and Huh7 cells under transfection of miR-448 mimics or control molecules via qRT-PCR. N=3. (B) The cell viability of HepG2 and Huh7. (C) Cells were checked after miR-448 mimic transfection via CCK-8 assay. N=3. Cell apoptosis was investigated by a FACS analysis in HepG2 $(\mathrm{D}, \mathrm{E})$ and Huh7 $(\mathrm{F}, \mathrm{G})$ with aberrant miR-448 expression. $(\mathrm{H})$ The statistical analysis for $(\mathrm{D}-\mathrm{G})$. N=3. ${ }^{* * *}, \mathrm{P}<0.001$.

especially glycolysis. Thus, the effect of glycolysis was examined in this study. The administration of miR-448 mimics decreased glucose uptake, lactate production, and ATP generation in both HepG2 and Huh7 cells (see Figure 4A-4C); Similarly, the knockdown of IGF-1R expression inhibited glucose uptake, lactate production and ATP generation in HepG2 and Huh7 cells (see Figure 4D-4F). However, the inhibition of miR-448 increased glucose uptake, lactate production, and ATP generation significantly in both HepG2 and Huh7 cells (see 
A

\begin{tabular}{|c|c|}
\hline 3' UT & '...AUUUGGGAUGUUCCUUUUUUCAAUAUGCAA... $3^{\prime}$ \\
\hline-4 & $\begin{array}{l}\text { UACCCUGUA-GGA----UGUAUACGUU } \\
\qquad||||::||||\end{array}$ \\
\hline$=1 R$ 3' UTR & AUUGCUGUAUUUUUCAAUAUG \\
\hline
\end{tabular}

B
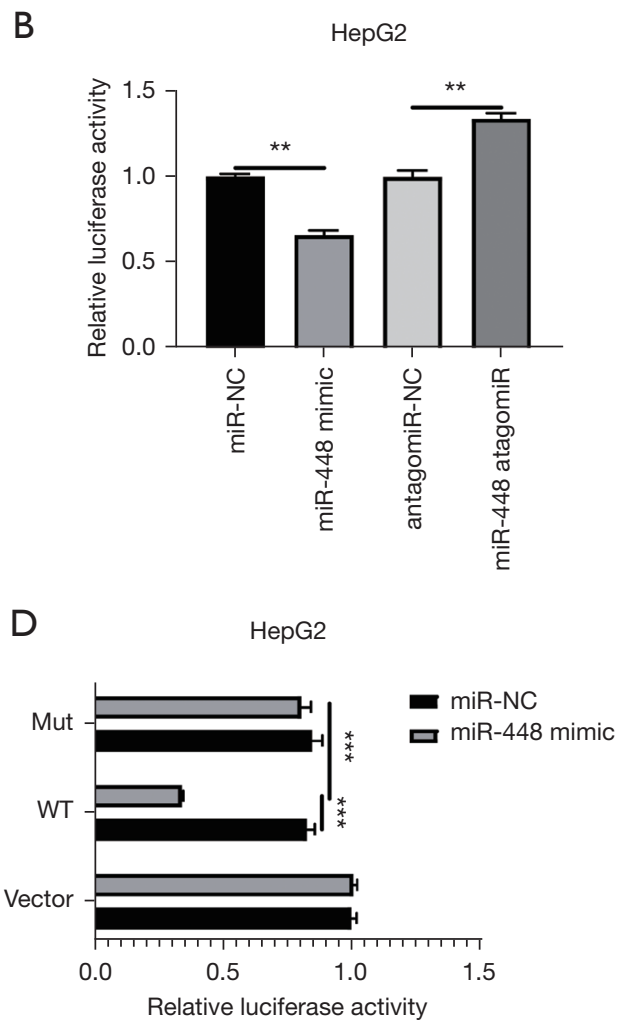

C<smiles>[TeH][TeH]</smiles>

$\mathrm{E}$

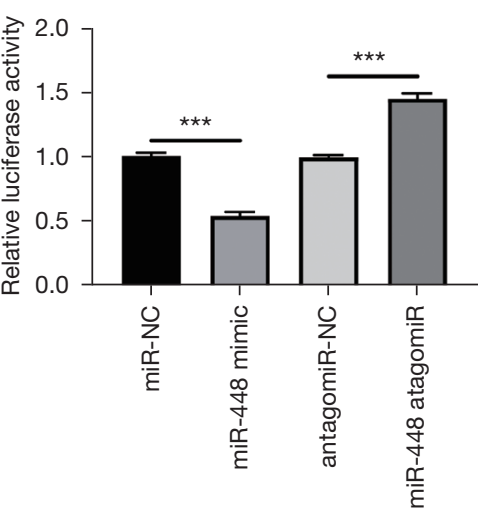

Huh7

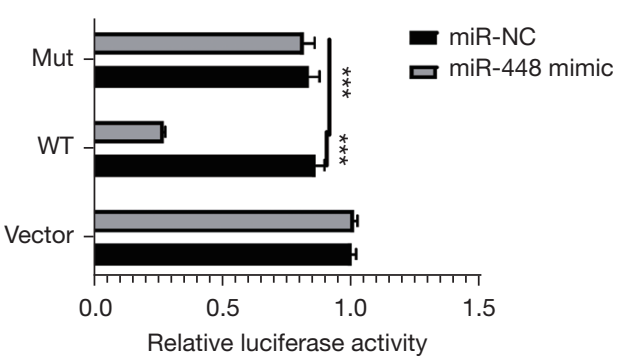

Figure 3 Insulin-like growth factor 1 receptor (IGF-1R) is a direct target of miR-448. (A) Schematic of the putative site showing miR448 bound to the IGF-1R 3'UTR. Luciferase reporter assay under the transfection of miR-448 mimic, miR-448 antagomir, and the corresponding control molecule in HepG2 (B) and Huh7 (C). N=3. HepG2 cells (D) and Huh7 cells (E) were transiently transfected with miR-448 mimics and control segments together with the pGL3-luci empty vector, a modified pGL3 vector containing wild-type IGF-1R 3'UTR or a mutated one. Luciferase reporter assay was performed to evaluate the relative luciferase activity. $\mathrm{N}=3$. **, $\mathrm{P}<0.01$; ***, $\mathrm{P}<0.001$.

Figure 4G-4I). In short, these findings suggest that miR-448 and IGF-1R play a role in regulating glycolysis in hepatoma cells.

\section{The regulatory effects of miR-448 on IGF-1R expression}

To examine the endogenous expression of miR-448 and IGF-1R, qRT-PCR was used to check their expression, and the results showed that miR-448 expression was relatively lower in Huh7 cells than in HepG2 cells, while the expression of IGF-1R was much higher in Huh7 cells than HepG2 cells (see Figure $5 A$ ). The transfection of the miR-448 mimics significantly decreased the expression of IGF-1R in both HepG2 and Huh7 cells. In addition, the inhibition by the antagomirs of miR-448 increased IGF1R expression significantly (see Figure 5B,5C). Again, the overexpression of miR-448 downregulated the IGF-1R protein level in HepG2 and Huh7 cells (see Figure 5D, 5E). In conclusion, miR-448 affects the expression of IGF-1R in hepatoma cells. 

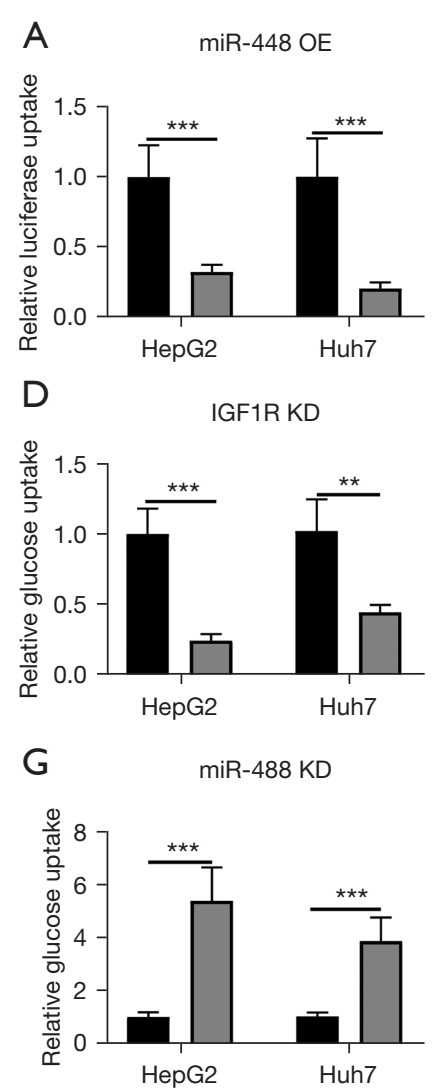
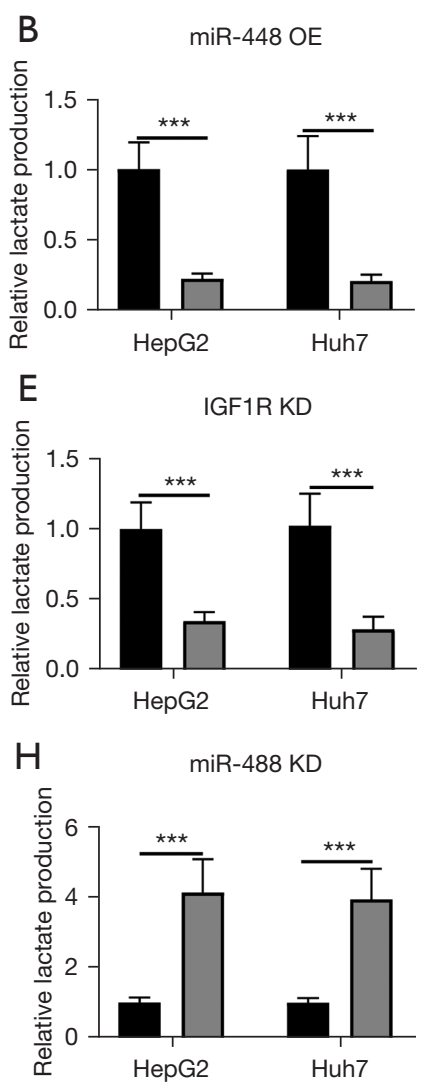
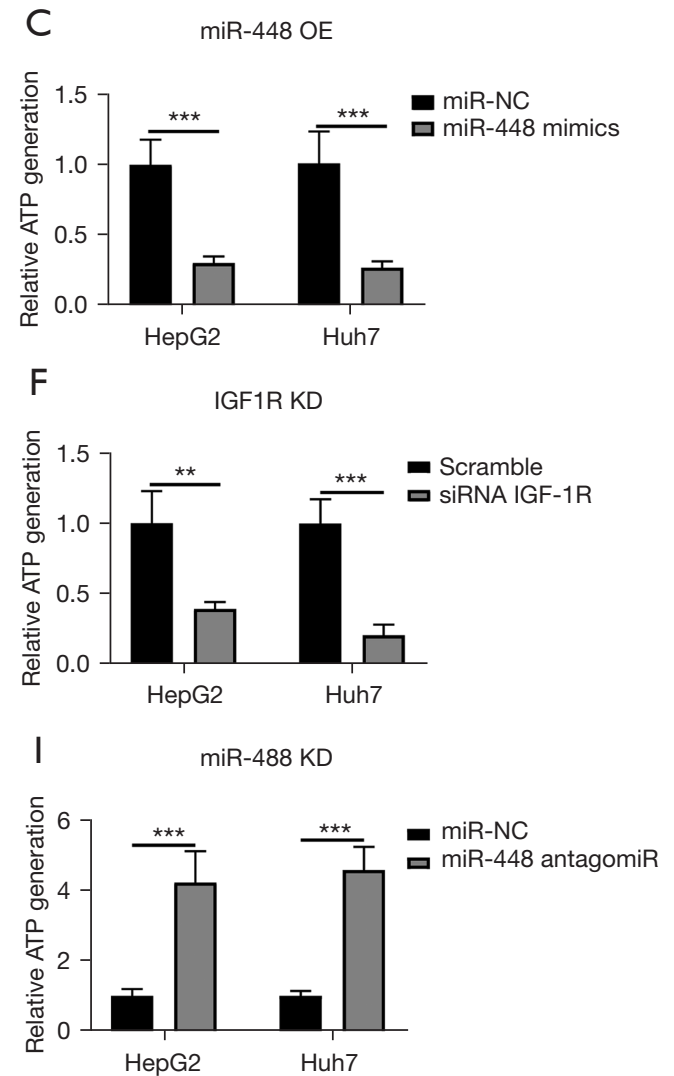

Figure 4 MiR-448 regulates glycolysis in hepatocellular carcinoma (HCC) cells. HepG2 and Huh7 cells were aberrantly transfected with miR-448 mimics and control constructs and then rate of glucose uptake (A), content of lactate production (B) and level of ATP generation (C) were measured. The rates of glucose uptake (D), content of lactate production (E), and level of ATP generation (F) were established under the transfection of insulin-like growth factor 1 receptor (IGF-1R) siRNA or control sequences. (G-I) The rates of glucose uptake, content of lactate production and ATP generation were measured in HepG2 and Huh7 cells. Endogenous miR-448 expression was down regulated by the transfection of the miR-448 antagomir. $\mathrm{N}=3$. ${ }^{* *}, \mathrm{P}<0.01 ;{ }^{* * *}, \mathrm{P}<0.001$.

\section{The enhanced expression of IGF-1R is associated with poor prognosis in HCC patients}

To clarify the clinical significance of IGF-1R in HCC, its expression in tumor tissues and adjacent liver tissues was examined by qRT-PCR. The results revealed that IGF-1R was more increased in the tumor tissues than the adjacent normal liver tissues (see Figure 6A). Additionally, on average, the $82 \mathrm{HCC}$ patients had an IGF-1R serum level of $65.2 \pm 19.8 \mathrm{ng} / \mathrm{mL}$, which was considerably higher than that of the healthy controls, who had an IGF-1R serum level of $29.2 \pm 13.7(\mathrm{P}<0.001$; see Table 2). To explore the relationship between IGF-1R and miR-448 in the
HCC patients, a Spearman correlation analysis was carried out, and the results revealed that miR-448 expression was reversely correlated to IGF-1R mRNA level (see Figure $6 B$ ). To determine the potential clinicopathological implications of IGF-1R expression, the K-M plotter database was used to examine the survival of the 64 HCC patients, and the results revealed a significant association between the elevated expression of IGF-1R and poor OS (see Figure 6C). However, the difference between the 2 groups in the DFS of HCC patients was not statistically significant (see Figure 6D). In summary, a high IGF-1R level is associated with poor OS in HCC patients. 
A

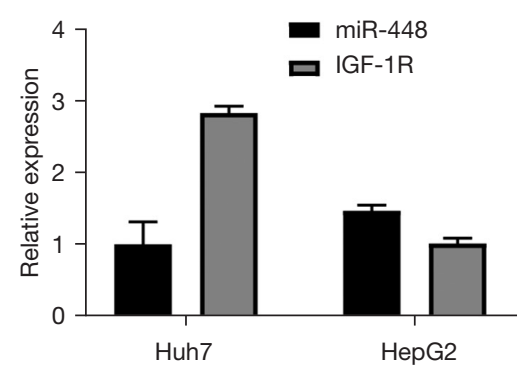

D
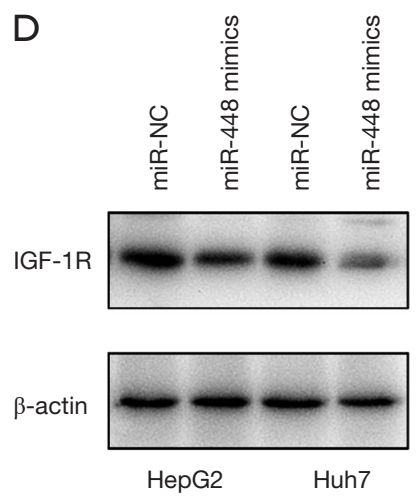

B

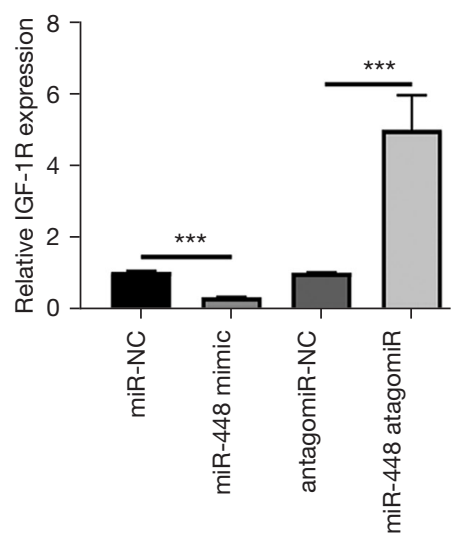

C

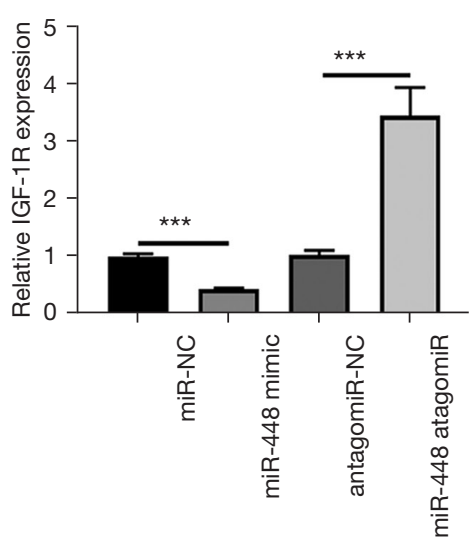

$E$

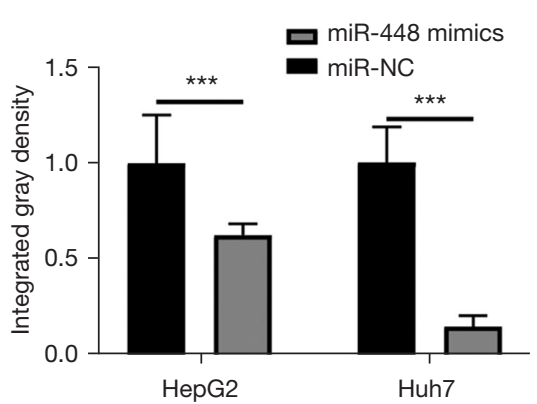

Figure 5 The regulatory effects of miR-448 on insulin-like growth factor 1 receptor (IGF-1R) expression. (A) The endogenous expression level of miR-448 and IGF-1R in HepG2 and Huh7 cells by qRT-PCR. The relative transcriptional expression levels of IGF-1R after being ectopically transfected with miR-448 mimics or control molecules in HepG2 (B) and Huh7 (C) cells. (D) The protein expression of IGF-1R in HepG2 and Huh7 cells under transfection with under transfection with miR-448 mimics and control molecules. (E) Relative gray density analysis of blots in (D). $\mathrm{N}=3 .{ }^{* * *}, \mathrm{P}<0.001$.

\section{Discussion}

HCC is notorious for its high mortality and morbidity rates. A lack of efficient early diagnosis biomarkers has worsened the situation, and HCC is now the 3rd leading cause of cancer-related deaths (3). Extensive research has shown that miRNA malfunction plays an important role in HCC progression $(7,9,22)$. Numerous studies have examined the functions of miR-448 in cancers, such as colon cancer (9), lung cancer (10), pituitary adenoma cells (11), pancreatic cancer (12), oral squamous cell carcinoma (13), breast cancer (14), bladder cancer (15), gastric cancer (16), and HCC (17). In HCC, miRNA is mostly regarded as a tumor suppressor.

In the present study, we examined the effects of miR-448 on hepatoma cell viability and glycolysis. The expression of miRNA-448 was markedly more decreased in tumor tissues than adjacent normal liver tissues. Additionally, the expression of IGF-1R was increased in HCC. To examine the regulatory mechanisms of miR-448 in HCC progression, the physical interaction between the 2 molecules was predicted by a bioinformatics analysis, and confirmed by dual-luciferase reporter assays. In addition, an inverse correlation was found between miR-448 and IGF-1R expression. Recent reports have shown that the low expression of miR-448 is associated with poor prognosis and prompts the epithelial mesenchymal transition process in HCC patients (17). The CCK-8 and FACS assays showed that the upregulation of miR-448 inhibits the cell viability of HCC cells, but increases their apoptosis in vitro.

IGF-1R is a key factor involved in the insulin pathway. Extensive research has shown that IGF-1R level is 
A

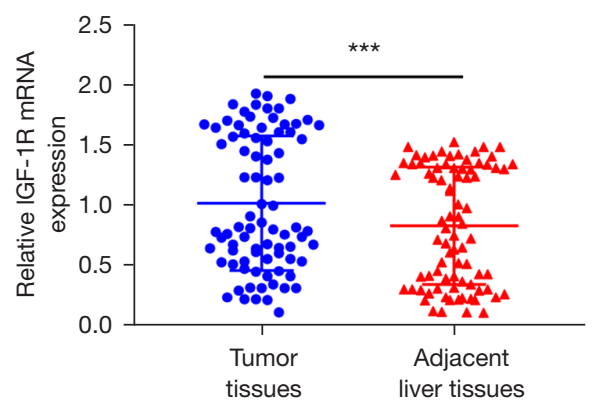

C

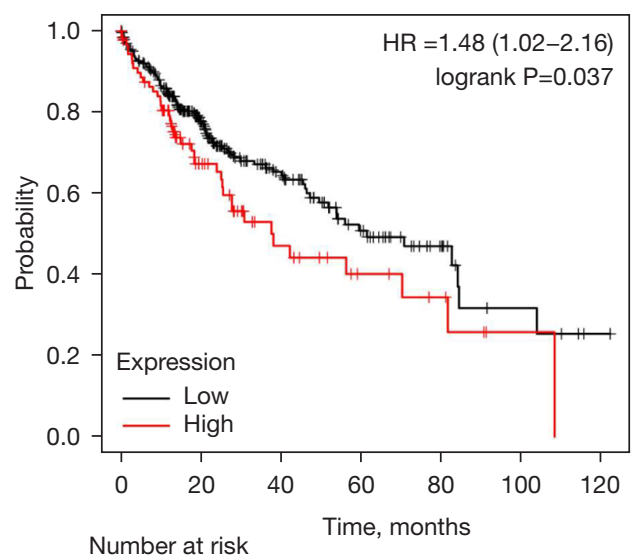

$\begin{array}{lccccccc}\text { Low } & 272 & 144 & 68 & 34 & 15 & 5 & 1\end{array}$
B

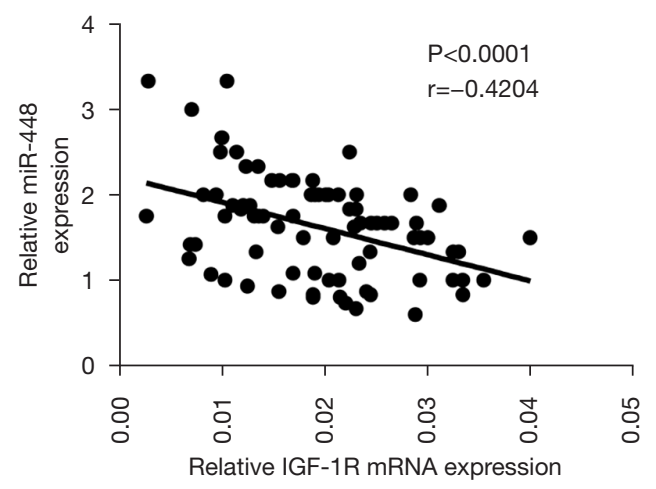

D IGF1R (3480)

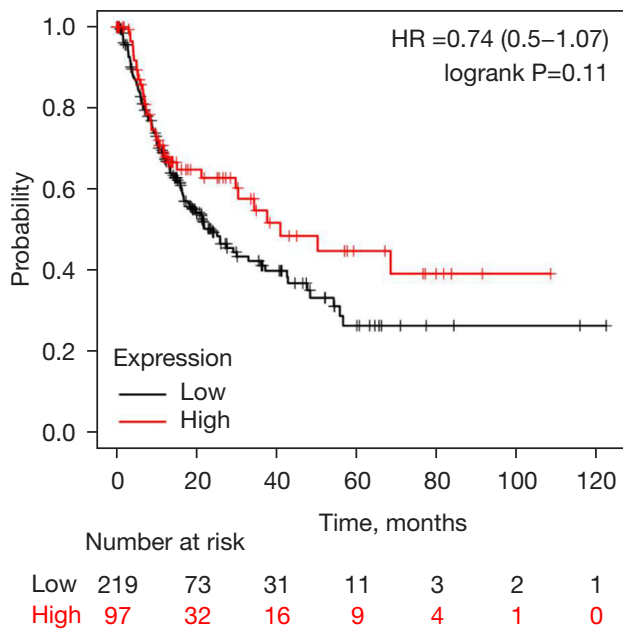

Figure 6 Upregulated insulin-like growth factor 1 receptor (IGF-1R) is associated with poor prognosis in hepatocellular carcinoma (HCC) patients. (A) Relative transcriptional expression of IGF-1R in HCC patients' tumor tissues and adjacent liver tissues. N=82. (B) Inverse correlation between miR-448 expression and IGF-1R mRNA levels in HCC tissues was tested by a Spearman correlation analysis. N=82. Correlations between IGF-1R and HCC patients' OS (C) and DFS (D), as compared by log-rank tests. ***, $\mathrm{P}<0.001$.

elevated in HCC patients, and is related to increased cell proliferation and poor prognosis (24). Further, IGF$1 \mathrm{R}$ has been reported to be involved in cancer cellrelated glycolysis which facilitates glucose uptake, lactate production, and ATP production (22). In our study, elevated IGF-1R expression was observed in HCC tumor tissues, and based on the clinical data provided by the K-M plotter database, it is significantly associated with poor OS. Additionally, the inhibition of IGF-1R expression decreases glucose uptake, lactate production, and ATP generation in HepG2 and Huh7 cells. Further, the aberrant expression of miR-448 decreases glycolysis activity in hepatoma cells, while knocking down the expression of miR-448 increased cell glycolysis.

Thus, miR-448, which is a critical oncomiR, is decreased in HCC patients and cells. Additionally, miR-448 and IGF$1 \mathrm{R}$ are inversely correlated and are both associated with tumor stage and size. Mechanistically, miR-448 inhibits hepatoma cell viability and glycolysis by suppressing IGF$1 \mathrm{R}$ by interacting with its 3'-UTR. However, it should be noted that our results were mainly derived from cell lines, and downstream molecular variations need to be further 
Table 2 Demographic data and clinical features of healthy control, and hepatocellular carcinoma groups

\begin{tabular}{lccc}
\hline Variable & $\begin{array}{c}\text { Healthy control } \\
(\mathrm{n}=82)\end{array}$ & $\begin{array}{c}\mathrm{HCC} \\
(\mathrm{n}=82)\end{array}$ & P value \\
\hline Sex (male/female) & $62 / 20$ & $68 / 14$ & \\
Age (years) & $48.4 \pm 12$ & $56.3 \pm 10.8$ & $<0.05$ \\
$\begin{array}{l}\text { Serum IGF-1R level } \\
\text { (ng/mL) }\end{array}$ & $29.2 \pm 13.7$ & $65.2 \pm 19.8$ & $<0.001$ \\
AST (U/L) & $18.8 \pm 4.9$ & $68.2 \pm 30.7$ & $<0.001$ \\
ALT (U/L) & $15.4 \pm 9.6$ & $66.3 \pm 30.4$ & $<0.001$ \\
AFP (ng/mL) & $1.2 \pm 0.6$ & $81.2 \pm 25.4$ & $<0.001$ \\
\hline
\end{tabular}

The significance of the difference between the two groups was tested using an analysis of variance (ANOVA) and the Bonferroni test to assess the individual differences between groups. HCC, hepatocellular carcinoma; AST, aspartate aminotransferase; ALT, alanine aminotransferase; AFP, alpha-fetoprotein.

investigated. In summary, miR-448 may be a potential biomarker for HCC diagnosis and a therapeutic target.

\section{Acknowledgments}

Funding: This study was supported by the National Natural Science Foundation of China (No. 81772645), the Science and Technology Plan Project of Suzhou (No. SLT201913), Beijing Xisike Clinical Oncology Research Foundation (No. Y-XD2019-227), the Research Grant of BeiGene (No. COMM-86), Horizontal Research Foundation of Soochow University (No. P142900221), and the Scientific Research Project of Nantong Municipal Health Commission (No. MA2021020).

\section{Footnote}

Reporting Checklist: The authors have completed the MDAR reporting checklist. Available at https://jgo.amegroups.com/ article/view/10.21037/jgo-22-90/rc

Data Sharing Statement: Available at https://jgo.amegroups. com/article/view/10.21037/jgo-22-90/dss

Conflicts of Interest: All authors have completed the ICMJE uniform disclosure form (available at https://jgo.amegroups. com/article/view/10.21037/jgo-22-90/coif). The authors have no conflicts of interest to declare.
Ethical Statement: The authors are accountable for all aspects of the work in ensuring that questions related to the accuracy or integrity of any part of the work are appropriately investigated and resolved. All the patients signed informed consent form. The present study was approved by the Ethics Committee of The Affiliated Tumor Hospital of Nantong University (ethic review No. LW2016-005), and the clinical investigation was conducted in accordance with the Declaration of Helsinki (as revised in 2013).

Open Access Statement: This is an Open Access article distributed in accordance with the Creative Commons Attribution-NonCommercial-NoDerivs 4.0 International License (CC BY-NC-ND 4.0), which permits the noncommercial replication and distribution of the article with the strict proviso that no changes or edits are made and the original work is properly cited (including links to both the formal publication through the relevant DOI and the license). See: https://creativecommons.org/licenses/by-nc-nd/4.0/.

\section{References}

1. Huang A, Yang XR, Chung WY, et al. Targeted therapy for hepatocellular carcinoma. Signal Transduct Target Ther 2020;5:146.

2. Dal Bo M, De Mattia E, Baboci L, et al. New insights into the pharmacological, immunological, and CAR-T-cell approaches in the treatment of hepatocellular carcinoma. Drug Resist Updat 2020;51:100702.

3. Frizziero M, McNamara MG, Lamarca A, et al. Current Translational and Clinical Challenges in Advanced Hepatocellular Carcinoma. Curr Med Chem 2020;27:4789-805.

4. Singal AG, Chen Y, Sridhar S, et al. Novel Application of Predictive Modeling: A Tailored Approach to Promoting HCC Surveillance in Patients With Cirrhosis. Clin Gastroenterol Hepatol 2021. [Epub ahead of print]. doi: 10.1016/j.cgh.2021.02.038.

5. Kulik L, Heimbach JK, Zaiem F, et al. Therapies for patients with hepatocellular carcinoma awaiting liver transplantation: A systematic review and meta-analysis. Hepatology 2018;67:381-400.

6. Roca Suarez AA, Testoni B, Zoulim F. HBV 2021: New therapeutic strategies against an old foe. Liver Int 2021;41 Suppl 1:15-23.

7. Bartel DP. MicroRNAs: genomics, biogenesis, mechanism, 
and function. Cell 2004;116:281-97.

8. Macfarlane LA, Murphy PR. MicroRNA: Biogenesis, Function and Role in Cancer. Curr Genomics 2010;11:537-61.

9. Guo JC, Yang YJ, Zhang JQ, et al. microRNA-448 inhibits stemness maintenance and self-renewal of hepatocellular carcinoma stem cells through the MAGEA6mediated AMPK signaling pathway. J Cell Physiol 2019;234:23461-74.

10. Qi H, Wang H, Pang D. miR-448 promotes progression of non-small-cell lung cancer via targeting SIRT1. Exp Ther Med 2019;18:1907-13.

11. He C, Yang J, Ding J, et al. MiR-448 targets BLC2 and inhibits the growth of pituitary adenoma cells. Biochem Cell Biol 2020;98:511-7.

12. Jin J, Wu Y, Zhou D, et al. miR-448 targets Rab2B and is pivotal in the suppression of pancreatic cancer. Oncol Rep 2018;40:1379-89.

13. Wei H, Yu K, Liu Y, et al. Tumor expression of miR-448 is a prognostic marker in oral squamous cell carcinoma. BMC Cancer 2020;20:756.

14. Ma P, Ni K, Ke J, et al. miR-448 inhibits the epithelialmesenchymal transition in breast cancer cells by directly targeting the E-cadherin repressor ZEB1/2. Exp Biol Med (Maywood) 2018;243:473-80.

15. Wang Y, Li LJ, Qiu MX, et al. Effects of paclitaxel combined with miR-448 on growth and proliferation of bladder cancer EJ cells. Eur Rev Med Pharmacol Sci 2018;22:3363-9.

Cite this article as: Wang Y, Chen X, Yao N, Gong J, Cao Y, Su X, Feng X, Tao M. MiR-448 suppresses cell proliferation and glycolysis of hepatocellular carcinoma through inhibiting IGF-1R expression. J Gastrointest Oncol 2022;13(1):355-367. doi: 10.21037/jgo-22-90
16. Wu X, Tang H, Liu G, et al. miR-448 suppressed gastric cancer proliferation and invasion by regulating ADAM10. Tumour Biol 2016;37:10545-51.

17. Zhu H, Zhou X, Ma C, et al. Low Expression of miR448 Induces EMT and Promotes Invasion by Regulating ROCK2 in Hepatocellular Carcinoma. Cell Physiol Biochem 2015;36:487-98.

18. Hanahan D, Weinberg RA. Hallmarks of cancer: the next generation. Cell 2011;144:646-74.

19. Vander Heiden MG, Cantley LC, Thompson CB. Understanding the Warburg effect: the metabolic requirements of cell proliferation. Science 2009;324:1029-33.

20. Cao J, Yan W, Ma X, et al. Insulin-like growth factor 2 mRNA-binding protein 2 - a potential link between type 2 diabetes mellitus and cancer. J Clin Endocrinol Metab 2021;106:2807-18.

21. Li J, Choi E, Yu H, et al. Structural basis of the activation of type 1 insulin-like growth factor receptor. Nat Commun 2019;10:4567.

22. Ngo MT, Jeng HY, Kuo YC, et al. The Role of IGF/IGF1R Signaling in Hepatocellular Carcinomas: StemnessRelated Properties and Drug Resistance. Int J Mol Sci 2021;22:1931.

23. Hakuno F, Takahashi SI. IGF1 receptor signaling pathways. J Mol Endocrinol 2018;61:T69-86.

24. Forbes BE, Blyth AJ, Wit JM. Disorders of IGFs and IGF-1R signaling pathways. Mol Cell Endocrinol 2020;518:111035. 